\title{
A Generalization of the $k$-Bonacci Sequence from Riordan Arrays
}

\author{
José L. Ramírez * \\ Departamento de Matemáticas \\ Universidad Sergio Arboleda \\ Bogotá, Colombia \\ josel.ramirez@ima.usergioarboleda.edu.co
}

\author{
Víctor F. Sirvent \\ Departamento de Matemáticas \\ Universidad Simón Bolívar \\ Caracas, Venezuela \\ vsirvent@usb.ve
}

Submitted: Aug 21, 2014; Accepted: Jan 15, 2015; Published: Feb 16, 2015

Mathematics Subject Classifications: 11B39, 05A19, 15A24.

\begin{abstract}
In this article, we introduce a family of weighted lattice paths, whose step set is $\left\{H=(1,0), V=(0,1), D_{1}=(1,1), \ldots, D_{m-1}=(1, m-1)\right\}$. Using these lattice paths, we define a family of Riordan arrays whose sum on the rising diagonal is the $k$-bonacci sequence. This construction generalizes the Pascal and Delannoy Riordan arrays, whose sum on the rising diagonal is the Fibonacci and tribonacci sequence, respectively. From this family of Riordan arrays we introduce a generalized $k$-bonacci polynomial sequence, and we give a lattice path combinatorial interpretation of these polynomials. In particular, we find a combinatorial interpretation of tribonacci and tribonacci-Lucas polynomials.
\end{abstract}

Keywords: Riordan arrays, $k$-bonacci sequence, lattice paths.

\section{Introduction}

A lattice path $\Gamma$ in the $x y$-plane with steps in a given set $S \subset \mathbb{Z}^{2}$ is a concatenation of directed steps of $S$, i.e., $\Gamma=s_{1} s_{2} \cdots s_{l}$, where $s_{i} \in S$ for $1 \leqslant i \leqslant l$. For any positive integer $n$, let $\mathbb{M}_{1}(n, k)$ denote the set of lattice paths from the point $(0,0)$ to the point $(k, n)$, with step set $S_{1}=\{H=(1,0), V=(0,1)\}$. Let $\mathcal{D}_{1}(n, k)$ be the number of lattice paths of $\mathbb{M}_{1}(n, k)$, i.e., $\mathcal{D}_{1}(n, k)=\left|\mathbb{M}_{1}(n, k)\right|$. It is well known that

$$
\mathcal{D}_{1}(n, k)=\left(\begin{array}{c}
n+k \\
n
\end{array}\right)=\left(\begin{array}{c}
n+k \\
k
\end{array}\right) .
$$

\footnotetext{
*The author was partially supported by Universidad Sergio Arboleda under grant no. DII-262.
} 
Let $\mathcal{H}_{1}$ be the infinite lower triangular array defined by $\mathcal{H}_{1}=\left[h_{n, k}^{(1)}\right]_{n, k \in \mathbb{N}}$, where

$$
h_{n, k}^{(1)}= \begin{cases}\mathcal{D}_{1}(n-k, k)=\left(\begin{array}{l}
n \\
k
\end{array}\right), & \text { if } n \geqslant k ; \\
0, & \text { if } n<k .\end{cases}
$$

Therefore, $\mathcal{H}_{1}$ is the Pascal matrix. This matrix has a lot of interesting properties, (see for instance $[2,5,6,7,12,23,24])$, one of them is the sum of the elements on the rising diagonal is the Fibonacci sequence $F_{n}$, (sequence A000045) ${ }^{1}$.

If we add a third step, $D_{1}=(1,1)$, we obtain Delannoy paths; among other references see $[1,9,10,13,22]$. We denote by $\mathbb{M}_{2}(n, k)$ the set of lattice paths from the point $(0,0)$ to the point $(k, n)$, with step set $S_{2}=\left\{H=(1,0), V=(0,1), D_{1}=(1,1)\right\}$. If $\mathcal{D}_{2}(n, k)=\left|\mathbb{M}_{2}(n, k)\right|$, then it is known that

$$
\mathcal{D}_{2}(n, k)=\sum_{i=0}^{k}\left(\begin{array}{c}
n \\
i
\end{array}\right)\left(\begin{array}{c}
n+k-i \\
n
\end{array}\right) .
$$

The sequence $\mathcal{D}_{2}(n, n)$ is called central Delannoy numbers (sequence A001850). Let $\mathcal{H}_{2}$ be the infinite lower triangular array defined by $\mathcal{H}_{2}=\left[h_{n, k}^{(2)}\right]_{n, k \in \mathbb{N}}$, where

$$
h_{n, k}^{(2)}= \begin{cases}\mathcal{D}_{2}(n-k, k), & \text { if } n \geqslant k ; \\ 0, & \text { if } n<k .\end{cases}
$$

This array is called Delannoy or tribonacci matrix. It satisfies that the sum of the elements on the rising diagonal is the tribonacci sequence $t_{n}$ (sequence A000073).

A generalized Delannoy number $\mathcal{D}_{2}^{*}(n, k)$ is the number of weighted lattice paths such that the steps $H, V$ and $D$ are labelled with weights $a, b$ and $c$, respectively. This kind of paths is called $(a, b, c)$-weighted paths [9]. The generalized Delannoy numbers are given by the following formula (cf. [15]):

$$
\mathcal{D}_{2}^{*}(n, k)=\sum_{i=0}^{k}\left(\begin{array}{l}
k \\
i
\end{array}\right)\left(\begin{array}{c}
n+k-i \\
k
\end{array}\right) a^{k-i} b^{n-i} c^{i} .
$$
where

Cheon et al. [9] defined the generalized Delannoy triangle $\mathcal{H}_{2}(a, b, c)=\left[d_{n, k}\right]_{n, k \in \mathbb{N}}$,

$$
d_{n, k}= \begin{cases}\mathcal{D}_{2}^{*}(n-k, k), & \text { if } n \geqslant k \\ 0, & \text { if } n<k\end{cases}
$$

\footnotetext{
${ }^{1}$ Many integer sequences and their properties are expounded on The On-Line Encyclopedia of Integer Sequences [20].
} 
and, they studied this array by means of Riordan arrays. The first few terms of this triangle are

$$
\mathcal{H}_{2}(a, b, c)=\left[\begin{array}{cccccc}
1 & 0 & 0 & 0 & 0 & \\
b & a & 0 & 0 & 0 & \\
b^{2} & c+2 a b & a^{2} & 0 & 0 & \\
b^{3} & b(2 c+3 a b) & a(2 c+3 a b) & a^{3} & 0 & \\
b^{4} & b^{2}(3 c+4 a b) & c^{2}+6 a b c+6 a^{2} b^{2} & a^{2}(3 c+4 a b) & a^{4} & \ldots \\
\vdots & & \vdots & & \vdots
\end{array}\right] .
$$

Additionally, they introduced a generalized Lucas polynomial sequence, and they gave a lattice path combinatorial interpretation for these polynomials. In particular, they found a combinatorial interpretation of the polynomials of Fibonacci, Lucas, Pell, PellLucas Chebyschev of first and second kind, among others. The study of lattice paths through Riordan arrays is not new, see $[18,21]$.

A natural questions is: What family of steps are required to find a Riordan array, such that the sum of the elements on its rising diagonal is the $k$-bonacci numbers $\mathcal{F}_{n}^{(k)}$ ? The $k$-bonacci numbers are defined by the recurrence

$$
\mathcal{F}_{n}^{(k)}=\mathcal{F}_{n-1}^{(k)}+\mathcal{F}_{n-2}^{(k)}+\cdots+\mathcal{F}_{n-k}^{(k)}, n \geqslant 1,
$$

with initial values $\mathcal{F}_{-1}^{(k)}=\mathcal{F}_{-2}^{(k)}=\cdots=\mathcal{F}_{-(k-1)}^{(k)}=0$ and $\mathcal{F}_{0}^{(k)}=1$. In particular, we obtain the Fibonacci sequence and tribonacci sequence, in the case $k=2,3$, respectively.

In this paper, we introduce a family of weighted lattice paths whose step set is

$$
S_{m}=\left\{H=(1,0), V=(0,1), D_{1}=(1,1), \ldots, D_{m-1}=(1, m-1)\right\},
$$

where each step is labelled with weights $a_{1}, a_{2}, \ldots, a_{m+1}$, respectively. From the number of these weighted lattice paths, we obtain a new family of Riordan arrays, such that the sum of the elements on its rising diagonal is the $k$-bonacci sequence. Moreover, we obtain a generalized $k$-bonacci polynomial sequence. The new family of weighted lattice paths leads us to a combinatorial interpretation for the generalized $k$-bonacci polynomial sequence. In particular, we study a generalization of the tribonacci polynomials and tribonacci-Lucas polynomials. We also show three combinatorial interpretations of the central tetrabonacci numbers.

\section{Riordan arrays}

A Riordan matrix $L=\left[l_{n, k}\right]_{n, k \in \mathbb{N}}$ is defined by a pair of generating functions $g(z)=$ $1+g_{1} z+g_{2} z^{2}+\cdots$ and $f(z)=f_{1} z+f_{2} z^{2}+\cdots$, where $f_{1} \neq 0$, such that the $k$-th column satisfies

$$
\sum_{n \geqslant 0} l_{n, k} z^{n}=g(z)(f(z))^{k}
$$


The first column being indexed by 0 . From the definition, follows

$$
l_{n, k}=\left[z^{n}\right] g(z)(f(z))^{k},
$$

where $\left[z^{n}\right]$ is the coefficient operator. The matrix corresponding to the pair $f(z), g(z)$ is denoted by $\mathcal{R}(g(z), f(z))$ or $(g(z), f(z))$. The product of two Riordan arrays $(g(z), f(z))$ and $(h(z), l(z))$ is defined by:

$$
(g(z), f(z)) *(h(z), l(z))=(g(z) h(f(z)), l(f(z))) .
$$

The set of all Riordan matrices is a group under the operator *, (cf. [19]). The identity element is $I=(1, z)$, and the inverse of $(g(z), f(z))$ is $(g(z), f(z))^{-1}=(1 /(g \circ \bar{f})(z), \bar{f}(z))$, where $\bar{f}(z)$ is the compositional inverse of $f(z)$.

Example 1. The Pascal matrix $\mathcal{H}_{1}$ and the Delannoy matrix $\mathcal{H}_{2}$ are given by the following Riordan arrays, respectively.

$$
\mathcal{H}_{1}=\left(\frac{1}{1-z}, \frac{z}{1-z}\right), \quad \mathcal{H}_{2}=\left(\frac{1}{1-z}, \frac{z(1+z)}{1-z}\right) .
$$

Example 2. ([9]) The generalized Delannoy array $\mathcal{H}_{2}(a, b, c)$ has a Riordan array expression given by

$$
\mathcal{H}_{2}(a, b, c)=\left(\frac{1}{1-b z}, z \frac{a+c z}{1-b z}\right)
$$

The following theorem is known as the fundamental theorem of Riordan arrays or summation property (cf. [21]).

Theorem 3. If $\left[l_{n, k}\right]_{n, k \in \mathbb{N}}=(g(z), f(z))$ is a Riordan matrix. Then for any sequence $\left\{h_{k}\right\}_{k \in \mathbb{N}}$

$$
\sum_{k=0}^{n} l_{n, k} h_{k}=\left[z^{n}\right] g(z) h(f(z)),
$$

where $h(z)$ is the generating function of the sequence $\left\{h_{k}\right\}_{k \in \mathbb{N}}$.

Let $\left[l_{n, k}\right]_{n, k \in \mathbb{N}}=(g(z), f(z))$ be a Riordan array, then the elements $\left\{l_{n-s k}: k \geqslant 0\right\}$ are called the d-diagonal of $(g(z), f(z))$ (cf. [21]).

\section{Generalized Tribonacci and Tribonacci-Lucas polynomial se- quence}

In this section, we defined a new kind of lattice paths: $(a, b, c, d)$-weighted paths. From the number of $(a, b, c, d)$-weighted lattice paths, we introduce a new infinite lower triangular array. Then, we study its properties and we obtain the generalized tribonacci and 
tribonacci-Lucas polynomial sequence.

Let $\mathbb{M}_{3}(n, k)$ denote the set of $(a, b, c, d)$-weighted paths from the point $(0,0)$ to the point $(k, n)$, with step set $S_{3}=\left\{H=(1,0), V=(0,1), D_{1}=(1,1), D_{2}=(1,2)\right\}$, where each step is labelled with weights $a, b, c$ and $d$, respectively. The weight of a weighted path is the product of the weights of all its steps in the weighted path and the length of a weighted path is the number of steps making up the path. For example, in Figure 1, we show a $(a, b, c, d)$-lattice path of length 5 and weight $a^{2} b c d$.

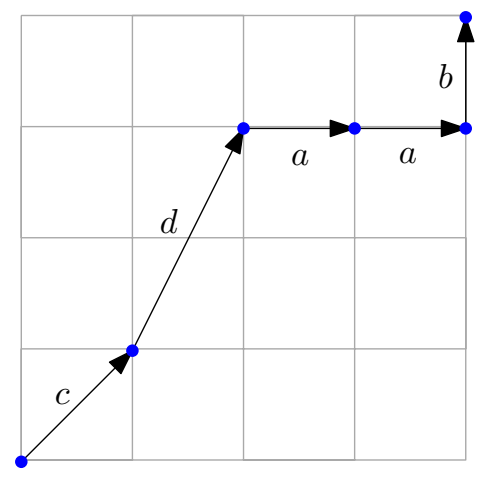

Figure 1: Example of $(a, b, c, d)$-weighted path.

The last step of any path from $\mathbb{M}_{3}(n, k)$ is one of $S_{3}$. Therefore, the number $\mathcal{D}_{3}(n, k):=$ $\left|\mathbb{M}_{3}(n, k)\right|$ satisfies the following four-term recurrence relation:

$$
\mathcal{D}_{3}(n, k)=a \mathcal{D}_{3}(n-1, k)+b \mathcal{D}_{3}(n, k-1)+c \mathcal{D}_{3}(n-1, k-1)+d \mathcal{D}_{3}(n-1, k-2),
$$

with $k \geqslant 2, n \geqslant 1$ and initial conditions $\mathcal{D}_{3}(0, k)=b^{k}$ and $\mathcal{D}_{3}(n, 0)=a^{n}$.

Theorem 4. The number of $(a, b, c, d)$-lattice paths is given by

$$
\begin{aligned}
\mathcal{D}_{3}(n, k) & =\sum_{j=0}^{n} \sum_{l=0}^{j}\left(\begin{array}{l}
n \\
j
\end{array}\right)\left(\begin{array}{l}
j \\
l
\end{array}\right)\left(\begin{array}{c}
n+k-2 j+l \\
k-2 j+l
\end{array}\right) a^{n-j} c^{l} d^{j-l} b^{k-2 j+l} \\
& =\sum_{j=0}^{n} \sum_{l=0}^{n-j}\left(\begin{array}{c}
n \\
j
\end{array}\right)\left(\begin{array}{c}
n-j \\
l
\end{array}\right)\left(\begin{array}{c}
k-n+2 j+l \\
n
\end{array}\right) a^{j} c^{l} d^{n-j-l} b^{k-2 n+2 j+l} .
\end{aligned}
$$

Proof. For $n \geqslant 1$, let

$$
\mathcal{W}_{n}^{(3)}(z):=\sum_{i=0}^{\infty} \mathcal{D}_{3}(n, i) z^{i} .
$$

Then by Equation (2), we obtain

$$
\mathcal{W}_{n}^{(3)}(z)=a \mathcal{W}_{n-1}^{(3)}(z)+b z \mathcal{W}_{n}^{(3)}(z)+c z \mathcal{W}_{n-1}^{(3)}(z)+d z^{2} \mathcal{W}_{n-1}^{(3)}(z)
$$


Then,

$$
\begin{gathered}
\mathcal{W}_{n}^{(3)}(z)=\left(\frac{a+c z+d z^{2}}{1-b z}\right) \mathcal{W}_{n-1}^{(3)}(z)=\left(\frac{a+c z+d z^{2}}{1-b z}\right)^{n} \mathcal{W}_{0}^{(3)}(z) \\
=\left(\frac{a+c z+d z^{2}}{1-b z}\right)^{n} \frac{1}{1-b z}=\frac{\left(a+c z+d z^{2}\right)^{n}}{(1-b z)^{n+1}}
\end{gathered}
$$

Therefore, by the binomial theorem

$$
\begin{aligned}
{\left[z^{k}\right] \mathcal{W}_{n}^{(3)} } & =\left[z^{k}\right]\left(\left(a+c z+d z^{2}\right)^{n}(1-b z)^{-(n+1)}\right) \\
& =\left[z^{k}\right]\left(\sum_{j=0}^{n} \sum_{l=0}^{j}\left(\begin{array}{c}
n \\
j
\end{array}\right)\left(\begin{array}{l}
j \\
l
\end{array}\right) a^{n-j}(c z)^{l}\left(d z^{2}\right)^{j-l} \sum_{i=0}^{\infty}\left(\begin{array}{c}
k+i \\
i
\end{array}\right)(b z)^{i}\right) \\
& =\left[z^{k}\right]\left(\sum_{j=0}^{n} \sum_{l=0}^{j} \sum_{i=0}^{\infty}\left(\begin{array}{c}
n \\
j
\end{array}\right)\left(\begin{array}{c}
j \\
l
\end{array}\right)\left(\begin{array}{c}
k+i \\
i
\end{array}\right) a^{n-j} c^{l} d^{j-l} b^{i} z^{2 j-l+i}\right) \\
& =\sum_{j=0}^{n} \sum_{l=0}^{j}\left(\begin{array}{c}
n \\
j
\end{array}\right)\left(\begin{array}{c}
j \\
l
\end{array}\right)\left(\begin{array}{c}
n+k-2 j+l \\
k-2 j+l
\end{array}\right) a^{n-j} c^{l} d^{j-l} b^{k-2 j+l} .
\end{aligned}
$$

Definition 5. Let $\mathcal{H}_{3}:=\mathcal{H}_{3}(a, b, c, d):=\left[d_{n, k}^{(3)}\right]_{n, k \in \mathbb{N}}$, where

$$
d_{n, k}^{(3)}= \begin{cases}\mathcal{D}_{3}(n-k, k), & \text { if } n \geqslant k ; \\ 0, & \text { if } n<k\end{cases}
$$

The first few terms of this triangle array are

$$
\mathcal{H}_{3}(a, b, c, d)=\left[\begin{array}{cccccc}
1 & 0 & 0 & 0 & 0 \\
b & a & 0 & 0 & 0 \\
b^{2} & 2 a b+c & a^{2} & 0 & 0 \\
b^{3} & 3 a b^{2}+2 c b+d & 3 b a^{2}+2 c a & a^{3} & 0 & \\
b^{4} & 4 a b^{3}+3 c b^{2}+2 d b & 6 a^{2} b^{2}+6 a c b+c^{2}+2 a d & 4 b a^{3}+3 c a^{2} & a^{4} & \ldots \\
\vdots & & \vdots & & \vdots &
\end{array}\right] .
$$

Theorem 6. The infinite triangular array $\mathcal{H}_{3}(a, b, c, d)$ has a Riordan array expression given by

$$
\mathcal{H}_{3}=\mathcal{H}_{3}(a, b, c, d)=\left(\frac{1}{1-b z}, z \frac{a+c z+d z^{2}}{1-b z}\right) .
$$

Proof. The proof runs like in Theorem 4, because

$$
\begin{aligned}
{\left[z^{n}\right] \mathcal{H}_{3}(a, b, c, d) } & =\left[z^{n}\right]\left(z^{k}\left(a+c z+d z^{2}\right)^{k}(1-b z)^{-(k+1)}\right) \\
& =\left[z^{n-k}\right]\left(\left(a+c z+d z^{2}\right)^{k}(1-b z)^{-(k+1)}\right) .
\end{aligned}
$$


Proposition 7. Let $\mathcal{A}_{3}(z)$ be the generating function for the rows sums of the Riordan array $\mathcal{H}_{3}$. Then

$$
\mathcal{A}_{3}(z)=\frac{1}{1-(a+b) z-c z^{2}-d z^{3}} .
$$

Proof. By applying Theorem 3 to the Riordan array $\mathcal{H}_{3}$ with the generating function $h(z)=(1-z)^{-1}$, we have

$$
\mathcal{A}_{3}(z)=\left(\frac{1}{1-b z}\right)\left(\frac{1}{1-\left(z \frac{a+c z+d z^{2}}{1-b z}\right)}\right)=\frac{1}{1-(a+b) z-c z^{2}-d z^{3}} .
$$

Each step weight $a, b, c, d$ can be considered as weighted functions $a=a(x), b=$ $b(x), c=c(x)$ and $d=d(x)$. Let $a+b=\bar{p}(x), c=\bar{q}(x), d=\bar{r}(x)$.

Let $\mathcal{F}_{n}^{(3)}(x)$ be the $n$-th row sum of $\mathcal{H}_{3}$, then from above proposition we obtain the generating function for $\mathcal{F}_{n}^{(3)}(x)$ :

$$
\mathcal{A}_{3}(z)=\sum_{i=0}^{\infty} \mathcal{F}_{i}^{(3)}(x) z^{i}=\frac{1}{1-\bar{p}(x) z-\bar{q}(x) z^{2}-\bar{r}(x) z^{3}} .
$$

The polynomials $\mathcal{F}_{n}^{(3)}(x)$ are called generalized tribonacci polynomials. The first few terms are

$$
1, p, p^{2}+q, p^{3}+2 p q+r, p^{4}+3 p^{2} q+2 p r+q^{2}, p^{5}+4 p^{3} q+3 p^{2} r+3 p q^{2}+2 q r, \ldots,
$$

where $q=\bar{q}(x), p=\bar{p}(x), r=\bar{r}(x)$. If $p=x^{2}, q=x$ and $r=1$, we get the tribonacci polynomials (cf. [16]).

Theorem 8. For all integer $n \geqslant 0$, and for any polynomials $\bar{p}(x), \bar{q}(x), \bar{r}(x)$

$$
\mathcal{F}_{n}^{(3)}(x)=\sum_{i=0}^{\left\lfloor\frac{n}{2}\right\rfloor} \sum_{j=0}^{i}\left(\begin{array}{c}
n-i-j \\
i
\end{array}\right)\left(\begin{array}{l}
i \\
j
\end{array}\right) \bar{q}^{i-j}(x) \bar{r}^{j}(x) \bar{p}^{n-2 i-j}(x) .
$$

Proof. The generating function of the sum of elements on the rising diagonal lines (1diagonal) in the generalized Delannoy triangle $\mathcal{H}_{2}(\bar{q}(x), \bar{p}(x), \bar{r}(x))$ is

$$
\left(\frac{1}{1-\bar{p}(x) z}\right)\left(\frac{1}{1-\left(z^{2} \frac{\bar{q}(x)+\bar{r}(x) z}{1-\bar{p}(x) z}\right)}\right)=\frac{1}{1-\bar{p}(x) z-\bar{q}(x) z^{2}-\bar{r}(x) z^{3}}=\mathcal{A}_{3}(z) .
$$

Then, by Equation (1)

$$
\mathcal{F}_{n}^{(3)}(x)=\sum_{i=0}^{\left\lfloor\frac{n-1}{2}\right\rfloor} D_{2}^{*}(n-1-i, i)=\sum_{i=0}^{\left\lfloor\frac{n-1}{2}\right\rfloor} \sum_{j=0}^{i}\left(\begin{array}{l}
i \\
j
\end{array}\right)\left(\begin{array}{c}
n-1-i-j \\
i
\end{array}\right) \bar{q}^{i-j}(x) \bar{r}^{j}(x) \bar{p}^{n-2 i-j-1}(x) .
$$


We give an alternative proof of Theorem 8 . Observe the equality

$$
\mathcal{A}_{3}(z)=\frac{1}{1-\bar{p}(x) z-\bar{q}(x) z^{2}-\bar{r}(x) z^{3}}=\frac{1}{1-\bar{q}(x) z^{2}} \cdot \frac{1}{1-w}
$$

where

$$
w=\frac{\bar{p}(x) z-\bar{r}(x) z^{3}}{1-\bar{q}(x) z^{2}}
$$

Therefore by Theorem 3, polynomials $\mathcal{F}_{n}^{(3)}(x)$ can be expressed by $n$-th sum of the rows of the following Riordan array

$$
\left(\frac{1}{1-\bar{q}(x) z^{2}}, \frac{\bar{p}(x) z-\bar{r}(x) z^{3}}{1-\bar{q}(x) z^{2}}\right)=\left[\begin{array}{cccccc}
1 & 0 & 0 & 0 & 0 & 0 \\
0 & p & 0 & 0 & 0 & 0 \\
q & 0 & p^{2} & 0 & 0 & 0 \\
0 & 2 p q-r & 0 & p^{3} & 0 & 0 \\
q^{2} & 0 & 3 p^{2} q-2 p r & 0 & p^{4} & 0 \\
0 & 3 p q^{2}-2 q r & 0 & 4 p^{3} q-3 p^{2} r & 0 & p^{5} \\
\vdots & & \vdots & & &
\end{array}\right],
$$

where $q=\bar{q}(x), p=\bar{p}(x), r=\bar{r}(x)$. So, the identity is clear.

Following the same ideas of Theorem 8 in [17], we obtain the following identity:

Proposition 9. For all integer $n \geqslant 0$, and for any polynomials $\bar{p}(x), \bar{q}(x), \bar{r}(x)$

$$
\mathcal{F}_{n}^{(3)}(x)=\sum_{0 \leqslant j \leqslant i \leqslant n}\left(\begin{array}{c}
n-i \\
i-j
\end{array}\right)\left(\begin{array}{c}
i-j \\
j
\end{array}\right) \bar{q}^{i-2 j}(x) \bar{r}^{j}(x) \bar{p}^{n-2 i+j}(x) .
$$

Theorem 10. For all integer $n \geqslant 3$, the polynomials $\mathcal{F}_{n}^{(3)}(x)$ satisfy the following recurrence:

$$
\mathcal{F}_{n}^{(3)}(x)=\bar{p}(x) \mathcal{F}_{n-1}^{(3)}(x)+\bar{q}(x) \mathcal{F}_{n-2}^{(3)}(x)+\bar{r}(x) \mathcal{F}_{n-3}^{(3)}(x),
$$

where $\mathcal{F}_{0}^{(3)}(x)=1, \mathcal{F}_{1}^{(3)}(x)=\bar{p}(x), \mathcal{F}_{2}^{(3)}(x)=\bar{p}^{2}(x)+\bar{q}(x)$.

Proof. The result follows from the equality

$$
\mathcal{A}_{3}(z)=\bar{p}(x) z \mathcal{A}_{3}(z)+\bar{q}(x) z^{2} \mathcal{A}_{3}(z)+\bar{r}(x) z^{3} \mathcal{A}_{3}(z) .
$$

We can write the polynomials $\mathcal{F}_{n}^{(3)}(x)$ as a Binet-like formula, i.e.,

$$
\begin{aligned}
\mathcal{F}_{n}^{(3)}(x)=\frac{\alpha(x)^{n+2}}{(\alpha(x)-\beta(x))(\alpha(x)-\gamma(x))} & \\
& \quad+\frac{\beta(x)^{n+2}}{(\beta(x)-\alpha(x))(\beta(x)-\gamma(x))}+\frac{\gamma(x)^{n+2}}{(\gamma(x)-\alpha(x))(\gamma(x)-\beta(x))},
\end{aligned}
$$

where $\alpha(x), \beta(x)$ and $\gamma(x)$ are the roots of the characteristic equation of (5). 
Definition 11. The generalized tribonacci-Lucas polynomials are defined by

$$
\mathcal{L}_{n}^{(3)}(x)=\alpha(x)^{n}+\beta(x)^{n}+\gamma(x)^{n}, \quad n \geqslant 0 .
$$

Note that

$$
\begin{aligned}
\mathcal{L}_{n}^{(3)}(x) & =\bar{p}(x) \mathcal{F}_{n-1}^{(3)}(x)+2 \bar{q}(x) \mathcal{F}_{n-2}^{(3)}(x)+3 \bar{r}(x) \mathcal{F}_{n-3}^{(3)}(x) \\
& =\mathcal{F}_{n}^{(3)}(x)+\bar{q}(x) \mathcal{F}_{n-2}^{(3)}(x)+2 \bar{r}(x) \mathcal{F}_{n-3}^{(3)}(x), n \geqslant 3, \\
& =\sum_{i=0}^{\left\lfloor\frac{n}{2}\right\rfloor} \sum_{j=0}^{i} \frac{n}{n-i-j}\left(\begin{array}{c}
i \\
j
\end{array}\right)\left(\begin{array}{c}
n-i-j \\
i
\end{array}\right) \bar{q}^{i-j}(x) \bar{r}^{j}(x) \bar{p}^{n-2 i-j}(x), n>i+j .
\end{aligned}
$$

The first few terms are

$3, p, p^{2}+2 q, p^{3}+3 p q+3 r, p^{4}+4 p^{2} q+4 p r+2 q^{2}, p^{5}+5 p^{3} q+5 p^{2} r+5 p q^{2}+5 q r, \ldots$, where $q=\bar{q}(x), p=\bar{p}(x), r=\bar{r}(x)$. If $p=q=r=1$, we obtain the tribonacci-Lucas sequence, (sequence A001644).

Proposition 12. The generating function of the tribonacci-Lucas polynomials is

$$
\mathcal{L}_{3}(z):=\sum_{i=0}^{\infty} \mathcal{L}_{i}^{(3)}(x) z^{i}=\frac{3-2 \bar{p}(x) z-\bar{q}(x) z^{2}}{1-\bar{p}(x) z-\bar{q}(x) z^{2}-\bar{r}(x) z^{3}} .
$$

Proof. From Equation (6), we have the following matrix equation:

$$
\mathcal{R}\left(1+\bar{q}(x) z^{2}+2 \bar{r}(x) z^{3}, z\right) \cdot\left[\mathcal{F}_{0}^{(3)}(x), \mathcal{F}_{1}^{(3)}(x), \ldots\right]^{T}=\left[1, \mathcal{L}_{1}^{(3)}(x), \mathcal{L}_{2}^{(3)}(x), \ldots\right]^{T}
$$

where $A^{T}$ is the transpose of the matrix $A$.

Then by applying Theorem 3 to the Riordan array $\mathcal{R}\left(1+\bar{q}(x) z^{2}+2 \bar{r}(x) z^{3}, z\right)$ with the generating function $\mathcal{A}_{3}(z)$, we get the generating function:

$$
\frac{1+\bar{q}(x) z^{2}+2 \bar{r}(x) z^{3}}{1-\bar{p}(x) z-\bar{q}(x) z^{2}-\bar{r}(x) z^{3}}
$$

Therefore,

$$
\mathcal{L}_{3}(z)=2+\frac{1+\bar{q}(x) z^{2}+2 \bar{r}(x) z^{3}}{1-\bar{p}(x) z-\bar{q}(x) z^{2}-\bar{r}(x) z^{3}}=\frac{3-2 \bar{p}(x) z-\bar{q}(x) z^{2}}{1-\bar{p}(x) z-\bar{q}(x) z^{2}-\bar{r}(x) z^{3}} .
$$

Theorem 13. Let $\bar{p}(x)=a x+b, \bar{q}(x)=c x$, and $\bar{r}(x)=d x$. Then

$$
\mathcal{F}_{n}^{(3)}(x)=\sum_{k=0}^{n}\left(\sum_{j=0}^{k} \sum_{l=0}^{j}\left(\begin{array}{l}
k \\
j
\end{array}\right)\left(\begin{array}{l}
j \\
l
\end{array}\right)\left(\begin{array}{c}
n-2 j+l \\
k
\end{array}\right) a^{k-j} b^{n-k-2 j+l} c^{l} d^{j-l}\right) x^{k} .
$$


Proof. By applying Theorem 3 to the Riordan array $\mathcal{H}_{3}$ with the generating function $h(z)=1 /(1-x z)$, we have

$$
\begin{aligned}
\sum_{k=0}^{n} \mathcal{D}_{3}(n-k, k) x^{k} & =\left[z^{n}\right]\left(\frac{1}{1-b z}\right)\left(\frac{1}{1-x z\left(\frac{a+c z+d z^{2}}{1-b z}\right)}\right) \\
& =\left[z^{n}\right]\left(\frac{1}{1-(b+a x) z-c x z^{2}-d x z^{3}}\right) \\
& =\left[z^{n}\right]\left(\frac{1}{1-\bar{p}(x) z-\bar{q}(x) z^{2}-\bar{r}(x) z^{3}}\right) .
\end{aligned}
$$

The coefficients of the above polynomial are the numbers of $(a, b, c, d)$-lattice paths.

\subsection{Combinatorial Interpretations}

The following theorem shows a combinatorial interpretation for the generalized tribonacci and tribonacci-Lucas polynomial sequences.

Theorem 14. For any polynomial $\mathcal{F}_{n}^{(3)}(x)$ and $\mathcal{L}_{n}^{(3)}(x)$, we have

(i) $\mathcal{F}_{n}^{(3)}(x)=\sum_{k=0}^{n} \omega_{n, k}^{(3)}(x), n \geqslant 0$

(ii) $\mathcal{L}_{n}^{(3)}(x)=\bar{p}(x) \sum_{k=0}^{n-1} \omega_{n-1, k}^{(3)}(x)+2 \bar{q}(x) \sum_{k=0}^{n-2} \omega_{n-2, k}^{(3)}(x)+3 \bar{r}(x) \sum_{k=0}^{n-3} \omega_{n-3, k}^{(3)}(x), \quad n \geqslant 3$,

where

$$
\omega_{n, k}^{(3)}(x):=\omega_{n, k}^{(3)}=h_{n, k}^{(3)}(x)=\mathcal{D}_{3}(n-k, k)
$$

is the sum of weights of $(a(x), b(x), c(x), d(x))$-weighted paths from $(0,0)$ to $(k, n-k)$ with step set

$$
S_{3}=\left\{H=(0,0), V=(0,1), D_{1}=(1,1), D_{2}=(1,2)\right\},
$$

such that $a(x)+b(x)=\bar{p}(x), c(x)=\bar{q}(x)$ and $d(x)=\bar{r}(x)$.

Proof. By definition, $\mathcal{F}_{n}^{(3)}(x)$ is the $n$-th row sum of the Riordan array $\mathcal{H}_{3}$. Then $(i)$ follows from Definition 5. Identity (ii) follows from Equation (6).

Example 15. The tribonacci numbers are defined by the recurrence relation:

$$
t_{0}=1, \quad t_{1}=1, \quad t_{2}=2, \quad t_{n+3}=t_{n+2}+t_{n+1}+t_{n}, \quad \text { for } n \geqslant 0 .
$$

The first few terms of the tribonacci number are 1, 1, 2, 4, 7, 13, 24, 44, 81, 149, 274,... (sequence A000073). Hoggatt and Bicknell [16] introduced tribonacci polynomials. The tribonacci polynomials $T_{n}(x)$ are defined by the recurrence relation

$$
\begin{aligned}
& T_{0}(x)=1, \quad T_{1}(x)=x^{2}, \quad T_{2}(x)=x^{4}+x, \\
& T_{n+3}(x)=x^{2} T_{n+2}(x)+x T_{n+1}(x)+T_{n}(x), \quad \text { for } n \geqslant 0 .
\end{aligned}
$$


Note that $T_{n}(1)=t_{n}$ for all integer positive $n$. The first few tribonacci polynomials are

$$
\begin{array}{ll}
T_{0}(x)=1, & T_{4}(x)=x^{8}+3 x^{5}+3 x^{2}, \\
T_{1}(x)=x^{2}, & T_{5}(x)=x^{10}+4 x^{7}+6 x^{4}+2 x, \\
T_{2}(x)=x^{4}+x, & T_{6}(x)=x^{12}+5 x^{9}+10 x^{6}+7 x^{3}+1, \\
T_{3}(x)=x^{6}+2 x^{3}+1, & T_{7}(x)=x^{14}+6 x^{11}+15 x^{8}+16 x^{5}+6 x^{2} .
\end{array}
$$

The generating function of the tribonacci polynomials is

$$
\sum_{n=0}^{\infty} T_{n}(x) z^{n}=\frac{1}{1-x^{2} z-x z^{2}-z^{3}} .
$$

If $a(x)=x^{2}, b(x)=0, c(x)=x$ and $d(x)=1$ in (3), we obtain the tribonacci polynomials from the row sums of the Riordan array $\mathcal{H}_{3}\left(x^{2}, 0, x, 1\right)$ :

$$
\left[\begin{array}{cccccccc}
1 & 0 & 0 & 0 & 0 & 0 & 0 & \\
0 & x^{2} & 0 & 0 & 0 & 0 & 0 & \\
0 & x & x^{4} & 0 & 0 & 0 & 0 \\
0 & 1 & 2 x^{3} & x^{6} & 0 & 0 & 0 \\
0 & 0 & 3 x^{2} & 3 x^{5} & x^{8} & 0 & 0 & \\
0 & 0 & 2 x & 6 x^{4} & 4 x^{7} & x^{10} & 0 & \\
0 & 0 & 1 & 7 x^{3} & 10 x^{6} & 5 x^{9} & x^{12} & \\
\vdots & & & \vdots & & & & \ddots
\end{array}\right] \cdot\left[\begin{array}{c}
1 \\
1 \\
1 \\
1 \\
1 \\
1 \\
1 \\
\vdots
\end{array}\right]=\left[\begin{array}{c}
T_{0}(x) \\
T_{1}(x) \\
T_{2}(x) \\
T_{3}(x) \\
T_{4}(x) \\
T_{5}(x) \\
T_{6}(x) \\
\vdots
\end{array}\right] .
$$

From Theorem 14- $(i)$, we obtain a combinatorial interpretation of $T_{n}(x)$. For example, $T_{4}(x)=x^{8}+3 x^{5}+3 x^{2}=\sum_{i=0}^{4} \omega_{4, i}^{(3)}$. Note that, $\omega_{4,0}^{(3)}=\omega_{4,1}^{(3)}=0, \omega_{4,2}^{(3)}=3 x^{2}, \omega_{4,3}^{(3)}=3 x^{5}$ and $\omega_{4,4}^{(3)}=x^{8}$. In Figure 2, we show the corresponding weighted lattice paths.

Example 16. The tribonacci-Lucas numbers are defined by the recurrence relation:

$$
k_{0}=3, \quad k_{1}=1, \quad k_{2}=3, \quad k_{n+3}=k_{n+2}+k_{n+1}+k_{n}, \quad \text { for } n \geqslant 0 .
$$

The first few terms of the tribonacci-Lucas numbers are 3, 1, 3, 7, 11, 21, 39, 71, 131, $241,443, \ldots$, (sequence A001644). The tribonacci-Lucas polynomials $K_{n}(x)$ are defined by the recurrence relation

$$
\begin{aligned}
& K_{0}(x)=3, \quad K_{1}(x)=x^{2}, \quad K_{2}(x)=x^{4}+2 x, \\
& K_{n+3}(x)=x^{2} K_{n+2}(x)+x K_{n+1}(x)+K_{n}(x), \quad n \geqslant 0 .
\end{aligned}
$$

Note that $K_{n}(1)=k_{n}$ for all integer positive $n$. The first few tribonacci-Lucas polynomials are

$$
\begin{aligned}
K_{0}(x) & =3, & K_{4}(x) & =x^{8}+4 x^{5}+6 x^{2}, \\
K_{1}(x) & =x^{2}, & K_{5}(x) & =x^{10}+5 x^{7}+10 x^{4}+5 x, \\
K_{2}(x) & =x^{4}+2 x, & K_{6}(x) & =x^{12}+6 x^{9}+15 x^{6}+14 x^{3}+3, \\
K_{3}(x) & =x^{6}+3 x^{3}+3, & K_{7}(x) & =x^{14}+7 x^{11}+21 x^{8}+28 x^{5}+14 x^{2} .
\end{aligned}
$$




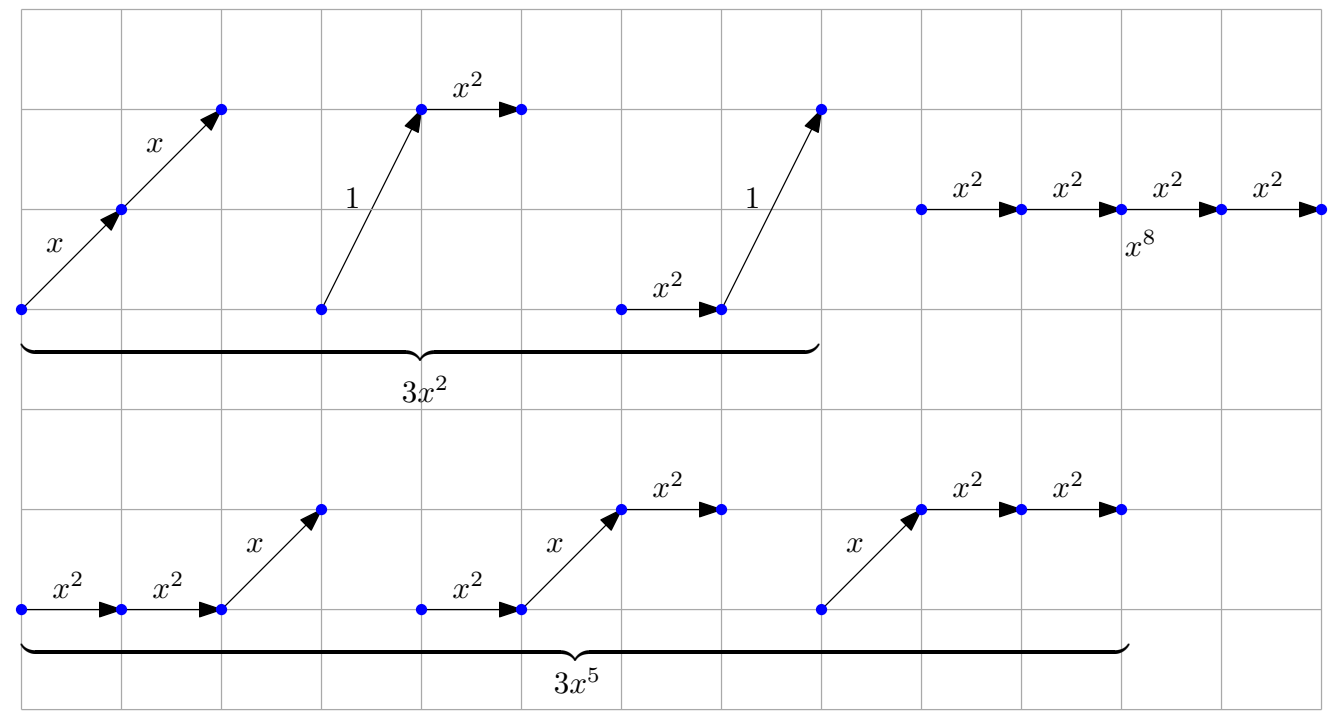

Figure 2: $\left(x^{2}, 0, x, 1\right)$-weighted paths and tribonacci polynomials.

Using standard techniques it can be shown that the generating function of the tribonacciLucas polynomials is

$$
\sum_{n=0}^{\infty} K_{n}(x) z^{n}=\frac{3-2 z x^{2}-z^{2} x}{1-x^{2} z-x z^{2}-z^{3}} .
$$

Since $\bar{p}(x)=a(x)+b(x)=x^{2}, \bar{q}(x)=c(x)=x$ and $\bar{r}(x)=d(x)=1$, we may choose $a(x)=x^{2}, b(x)=0, c(x)=x$ and $d(x)=1$. From Theorem 14-(ii), we obtain a combinatorial interpretation of $K_{n}(x)$. For example,

$$
K_{3}(x)=x^{6}+3 x^{3}+3=x^{2} \sum_{i=0}^{2} \omega_{2, i}^{(3)}+2 x \sum_{i=0}^{1} \omega_{1, i}^{(3)}+3 \omega_{0,0}^{(3)} .
$$

Note that $\omega_{2,0}^{(3)}=0, \omega_{2,1}^{(3)}=x, \omega_{2,0}^{(3)}=x^{4}, \omega_{1,0}^{(3)}=0, \omega_{1,1}^{(3)}=x^{2}$ and $\omega_{0,0}^{(3)}=1$. In Figure 3, we show the corresponding weighted lattice paths.

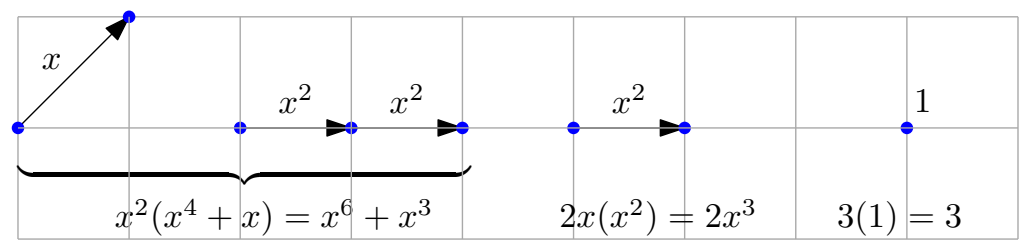

Figure 3: $\left(x^{2}, 0, x, 1\right)$-weighted paths, and tribonacc-Lucas polynomials. 


\subsection{The diagonal of $\mathcal{D}_{3}$}

Let the Riordan array $\mathcal{H}_{3}(1,1,1,1)$. The first few terms of this array are

$$
\mathcal{H}_{3}(1,1,1,1)=\left[\begin{array}{ccccccc}
1 & 0 & 0 & 0 & 0 & 0 & \\
1 & 1 & 0 & 0 & 0 & 0 & \\
1 & 3 & 1 & 0 & 0 & 0 & \\
1 & 6 & 5 & 1 & 0 & 0 & \\
1 & 9 & 15 & 7 & 1 & 0 & \\
1 & 12 & 33 & 28 & 9 & 1 & \\
\vdots & & \vdots & & & & \ddots
\end{array}\right]
$$

From Theorem 6 of [3], we have that the generating function of the central sequence $\left\{d_{2 n, n}^{(3)}\right\}_{n \in \mathbb{N}}=\{1,3,15,81,459,2673, \ldots\}$ is

$$
\sum_{n=0}^{\infty} d_{2 n, n}^{(3)} z^{n}=\sum_{n=0}^{\infty} \mathcal{D}_{3}(n, n) z^{n}=\frac{1}{\sqrt{1-6 z-3 z^{2}}}
$$

On the other hand, a Motzkin path of length $n$ is a lattice path of $\mathbb{Z} \times \mathbb{Z}$ running from $(0,0)$ to $(n, 0)$ that never passes below the $x$-axis and whose permitted steps are the up diagonal step $U=(1,1)$, the down diagonal step $D=(1,-1)$ and the horizontal step $H=(1,0)$, called rise, fall and level step, respectively. The number of Motzkin paths of length $n$ is the $n$-th Motzkin number $m_{n}$, (sequence A001006). Many other examples of bijections between Motzkin numbers and others combinatorial objects can be found in [4]. A Grand Motzkin path of length $n$ is a Motzkin path without the condition that never passes below the $x$-axis. The number of Grand Motzkin paths of length $n$ is the $n$-th Grand Motzkin number $g_{n}$, sequence A002426. A $a^{H} b^{U}$-Motzkin path is a Motzkin path such that each horizontal step is colored with one of $a$ specific colors and each up diagonal step is colored with one of $b$ specific colors. The number of $a^{H} b^{U}$-Motzkin paths of length $n$ is the $n$-th $a^{H} b^{U}$-Motzkin number $m_{n}^{(a, b)}$. Analogously, we have $a^{H} b^{U}$-Grand Motzkin paths, the number of $a^{H} b^{U}$-Grand Motzkin paths of length $n$ is denoted by $g_{n}^{(a, b)}$.

From Theorem 1 of [8], we have

$$
\sum_{n=0}^{\infty} g_{n}^{(3,3)} z^{n}=\frac{1}{\sqrt{1-6 z-3 z^{2}}}
$$

Then, we get the following corollary.

Corollary 17. The number of $3^{H} 3^{U}$-Grand Motzkin path is equal to the number of $(1,1,1,1)$-weighted paths from the point $(0,0)$ to the point $(n, n)$, i.e.,

$$
g_{n}^{(3,3)}=\mathcal{D}_{3}(n, n) .
$$


Let $S(n, k)$ be the number of lattice paths from $(0,0)$ to $(n, k)$ in the plane $\mathbb{Z} \times \mathbb{N}$ with set of steps $S_{3}^{\prime}=\{H=(1,0), V=(0,1), D=(1,1), L=(-1,1)\}$. In [11], Dziemiańczuk shows that

$$
\sum_{n=0}^{\infty} S(0, n) z^{n}=\frac{1}{\sqrt{1-6 z-3 z^{2}}} .
$$

Therefore

$$
S(0, n)=g_{n}^{(3,3)}=\mathcal{D}_{3}(n, n)
$$

Moreover,

$$
(S(i-2 j, j))_{i, j \geqslant 0}=\mathcal{H}_{3}(1,1,1,1)
$$

then $S(i-2 j, j)=\mathcal{D}_{3}(i-j, j)$.

\section{Riordan Arrays and Generalized $k$-bonacci numbers}

In this section, we generalize the results of the above section. We introduce a new family of weighted lattice paths, the $\vec{A}_{s}$-weighted paths.

Let $\vec{A}_{s}=\left(a_{1}, a_{2}, \ldots, a_{s}\right)$ be a vector of weights. Then, we denote by $\mathbb{M}_{m}(n, k)$ the set of $\vec{A}_{m+1}$-weighted paths from the point $(0,0)$ to the point $(k, n)$, with step set

$$
S_{m}=\left\{H=(1,0), V=(0,1), D_{1}=(1,1), \ldots, D_{m-1}=(1, m-1)\right\},
$$

where each step is labelled with weights $a_{1}, a_{2}, \ldots, a_{m+1}$, respectively. Let $\mathcal{D}_{m}(n, k)=$ $\left|\mathbb{M}_{m}(n, k)\right|$. Note that if $m=2,3$ we obtain the generalized Delannoy paths and the $(a, b, c, d)$-weighted paths, respectively.

Lemma 18. The numbers $\mathcal{D}_{m}(n, k)$ satisfy the following $(m+1)$-term recurrence relation

$$
\mathcal{D}_{m}(n, k)=a_{1} \mathcal{D}_{m}(n-1, k)+a_{2} \mathcal{D}_{m}(n, k-1)+\sum_{j=1}^{m-1} a_{j+2} \mathcal{D}_{m}(n-1, k-j),
$$

with $k \geqslant m-1, n \geqslant 1$ and initial conditions $\mathcal{D}_{m}(0, k)=a_{2}^{k}$ and $\mathcal{D}_{m}(n, 0)=a_{1}^{n}$.

Theorem 19. The number of $\vec{A}_{m+1}$-lattice paths is given by

$$
\begin{aligned}
\mathcal{D}_{m}(n, k)=\sum_{j_{1}=0}^{n} \sum_{j_{2}=0}^{n-j_{1}} \cdots \sum_{j_{m-1}=0}^{n-\sum_{j=1}^{m-2} j_{i}}\left(\begin{array}{c}
n \\
j_{1}
\end{array}\right)\left(\begin{array}{c}
n-j_{1} \\
j_{2}
\end{array}\right) \cdots\left(\begin{array}{c}
n-\sum_{j=1}^{m-2} j_{i} \\
j_{m-1}
\end{array}\right) \\
\times\left(\begin{array}{c}
n+k-u \\
n
\end{array}\right) a_{1}^{j_{1}} a_{3}^{j_{2}} \cdots a_{m}^{j_{m}-1} a_{m+1}^{n-\sum_{i=1}^{m-1} j_{i}} a_{2}^{k-u}
\end{aligned}
$$

where

$$
u=(m-1)\left(n-j_{1}\right)+\sum_{i=2}^{m-1}(i-m) j_{i}
$$


Proof. For $n \geqslant 1$, let

$$
\mathcal{W}_{n}^{(m)}(z):=\sum_{i=0}^{\infty} \mathcal{D}_{m}(n, i) z^{i}
$$

Then from Equation (9), we have

$$
\mathcal{W}_{n}^{(m)}(z)=a_{1} \mathcal{W}_{n-1}^{(m)}(z)+a_{2} z \mathcal{W}_{n}^{(m)}(z)+\sum_{j=1}^{m-1} a_{j+2} z^{j} \mathcal{W}_{n-1}^{(m)}(z)
$$

Then,

$$
\begin{gathered}
\mathcal{W}_{n}^{(m)}(z)=\left(\frac{a_{1}+\sum_{j=1}^{m-1} a_{j+2} z^{j}}{1-a_{2} z}\right) \mathcal{W}_{n-1}^{(m)}(z)=\left(\frac{a_{1}+\sum_{j=1}^{m-1} a_{j+2} z^{j}}{1-a_{2} z}\right)^{n} \mathcal{W}_{0}^{(m)}(z) \\
=\left(\frac{a_{1}+\sum_{j=1}^{m-1} a_{j+2} z^{j}}{1-a_{2} z}\right)^{n} \frac{1}{1-a_{2} z}=\frac{\left(a_{1}+\sum_{j=1}^{m-1} a_{j+2} z^{j}\right)^{n}}{\left(1-a_{2} z\right)^{n+1}} .
\end{gathered}
$$

Therefore, by the binomial theorem

$$
\begin{aligned}
{\left[z^{k}\right] \mathcal{W}_{n}^{(m)} } & =\left[z^{k}\right]\left(\left(a_{1}+\sum_{j=1}^{m-1} a_{j+2} z^{j}\right)^{n}\left(1-a_{2} z\right)^{-(n+1)}\right) \\
& =\left[z^{k}\right]\left(\sum_{j_{1}=0}^{n} \sum_{j_{2}=0}^{n-j_{1}} \ldots \sum_{j_{m-1}=0}^{n-\sum_{i=1}^{m-1} j_{i}}\left(\begin{array}{c}
n \\
j_{1}
\end{array}\right)\left(\begin{array}{c}
n-j_{1} \\
j_{2}
\end{array}\right) \cdots\left(\begin{array}{c}
n-\sum_{i=1}^{m-2} j_{i} \\
j_{m-1}
\end{array}\right)\right. \\
& \left.\times a_{1}^{j_{1}}\left(a_{3} z\right)^{j_{2}} \cdots\left(a_{m} z^{m-2}\right)^{j_{m-2}}\left(a_{m+1} z^{m-1}\right)^{n-\sum_{i=1}^{m-1} j_{i}} \sum_{l=0}^{\infty}\left(\begin{array}{c}
n+l \\
n
\end{array}\right)\left(a_{2} z\right)^{l}\right) \\
& =\left[z^{k}\right]\left(\sum_{j_{1}=0}^{n} \sum_{j_{2}=0}^{n-j_{1}} \ldots \sum_{j_{m-1}=0}^{n-\sum_{i=1}^{m-1} j_{i}} \sum_{l=0}^{\infty}\left(\begin{array}{c}
n \\
j_{1}
\end{array}\right)\left(\begin{array}{c}
n-j_{1} \\
j_{2}
\end{array}\right) \cdots\left(\begin{array}{c}
n-\sum_{i=1}^{m-2} j_{i} \\
j_{m-1}
\end{array}\right)\left(\begin{array}{c}
n+l \\
n
\end{array}\right)\right. \\
& \left.\times a_{1}^{j_{1}} a_{3}^{j_{2}} \cdots a_{m}^{j_{m-2}} a_{m+1}^{n-\sum_{i=1}^{m-1} j_{i}} a_{2}^{l} z^{u+l}\right) \\
& =\sum_{j_{1}=0}^{n} \sum_{j_{2}=0}^{n-j_{1}} \ldots \sum_{n-\sum_{i=1}^{m-1} j_{i}}^{n}\left(\begin{array}{c}
n \\
j_{1}
\end{array}\right)\left(\begin{array}{c}
n-j_{1} \\
j_{2}
\end{array}\right) \cdots\left(\begin{array}{c}
n-\sum_{i=1}^{m-2} j_{i} \\
j_{m-1}
\end{array}\right)\left(\begin{array}{c}
n+k-u \\
i
\end{array}\right) \\
& \times a_{1}^{j_{1}} a_{3}^{j_{2}} \cdots a_{m}^{j_{m-2}} a_{m+1}^{n-\sum_{i=1}^{m-1} j_{i}} a_{2}^{k-u} .
\end{aligned}
$$

Definition 20. Let $\mathcal{H}_{m}:=\mathcal{H}_{m}\left(a_{1}, a_{2}, \ldots, a_{m+1}\right):=\left[d_{n, k}^{(m)}\right]_{n, k \in \mathbb{N}}$, where

$$
d_{n, k}^{(m)}= \begin{cases}\mathcal{D}_{m}(n-k, k), & \text { if } n \geqslant k \\ 0, & \text { if } n<k\end{cases}
$$


Theorem 21. The infinite triangular array $\mathcal{H}_{m}$ has a Riordan array expression given by

$$
\mathcal{H}_{m}=\left(\frac{1}{1-a_{2} z}, z \frac{a_{1}+a_{3} z+a_{4} z^{2}+\cdots+a_{m+1} z^{m-1}}{1-a_{2} z}\right) .
$$

Proof. It is clear from Theorem 19.

For example, the first few terms of the Riordan array $\mathcal{H}_{4}(a, b, c, d, e)$ are

$$
\mathcal{H}_{4}=\left[\begin{array}{ccccc}
1 & 0 & 0 & 0 & 0 \\
b & a & 0 & 0 & 0 \\
b^{2} & 2 a b+c & a^{2} & 0 & 0 \\
b^{3} & 3 a b^{2}+2 c b+d & 3 b a^{2}+2 c a & a^{3} & 0 \\
b^{4} & 4 a b^{3}+3 c b^{2}+2 d b+e & 6 a^{2} b^{2}+6 a c b+c^{2}+2 a d & 4 b a^{3}+3 c a^{2} & a^{4} \\
\vdots & & \vdots & & \vdots
\end{array}\right] .
$$

Proposition 22. Let $\mathcal{A}_{m}(z)$ be the generating function for the rows sums of the Riordan array $\mathcal{H}_{m}$. Then

$$
\mathcal{A}_{m}(z)=\frac{1}{1-\left(a_{1}+a_{2}\right) z-a_{3} z^{2}-\cdots-a_{m+1} z^{m}} .
$$

Proof. The proof runs like in Proposition 7.

Let $\mathcal{F}_{n}^{(k)}(x)$ be the $n$-th row sum of $\mathcal{H}_{k}$, then from above proposition we obtain the generating function for $\mathcal{F}_{n}^{(k)}(x)$ :

$$
\mathcal{A}_{k}(z)=\sum_{i=0}^{\infty} \mathcal{F}_{i}^{(k)}(x) z^{i}=\frac{1}{1-\bar{p}_{1}(x) z-\bar{p}_{2}(x) z^{2}-\cdots-\bar{p}_{k}(x) z^{k}},
$$

where $\bar{p}_{1}(x)=a_{1}+a_{2}$, and $\bar{p}_{j}(x)=a_{j+1}, j=2, \ldots, k$. The polynomials $\mathcal{F}_{n}^{(k)}(x)$ are called generalized $k$-bonacci polynomials.

For example, if $k=4$ we obtain the tetrabonacci polynomials. The first few terms of the tetrabonacci polynomials sequence are

$$
1, p_{1}, p_{1}^{2}+p_{2}, p_{1}^{3}+2 p_{2} p_{1}+p_{3}, p_{1}^{4}+3 p_{2} p_{1}^{2}+2 p_{3} p_{1}+p_{2}^{2}+p_{4}, \ldots,
$$

where $p_{i}=\bar{p}_{i}(x), i=1,2,3,4$.

The sum on the rising diagonal in the Pascal triangle and in the tribonacci triangle are the Fibonacci numbers and tribonacci numbers, respectively. In general, we have the following proposition.

Proposition 23. The $k$-bonacci triangle $\mathcal{T}_{k}$ defined by the following Riordan array

$$
\mathcal{T}_{k}=\left(\frac{1}{1-z}, z \frac{1+z+\cdots+z^{k-1}}{1-z}\right)
$$

satisfies that the sum on the rising diagonal is the $k$-bonacci sequence $\mathcal{F}_{n}^{(k)}$, where $\mathcal{F}_{n}^{(k)}=$ $\mathcal{F}_{n-1}^{(k)}+\mathcal{F}_{n-2}^{(k)}+\cdots+\mathcal{F}_{n-k}^{(k)}$. 
From Theorem 3 and Theorem 19, we have the following identity.

Theorem 24. For all integer $n \geqslant 0$, and for any polynomials $\bar{p}_{i}(x)=p(x), i=1,2, \ldots, k+$ 1 ,

$$
\begin{aligned}
& \mathcal{F}_{n}^{(k)}(x)=\sum_{i=0}^{\left\lfloor\frac{n-1}{2}\right\rfloor} \mathcal{D}_{k}(n-1-s, s) \\
&=\sum_{i=0}^{\left\lfloor\frac{n-1}{2}\right\rfloor} \sum_{j_{1}=0}^{s} \sum_{j_{2}=0}^{s-j_{1}} \cdots \sum_{j_{k-1}=0}^{s-\sum_{j=1}^{k-2} j_{i}}\left(\begin{array}{c}
s \\
j_{1}
\end{array}\right)\left(\begin{array}{c}
s-j_{1} \\
j_{2}
\end{array}\right) \cdots\left(\begin{array}{c}
s-\sum_{j=1}^{k-2} j_{i} \\
j_{k-1}
\end{array}\right) \\
& \times\left(\begin{array}{c}
n-u+1 \\
s
\end{array}\right) p_{1}^{j_{1}} p_{3}^{j_{2}} \cdots p_{k}^{j_{k-1}} p_{k+1}^{s-\sum_{i=1}^{k-1} j_{i}} p_{2}^{n-u-s-1},
\end{aligned}
$$

where

$$
u=(k-1)\left(s-j_{1}\right)+\sum_{i=2}^{k-1}(i-k) j_{i} .
$$

Theorem 25. The polynomials $\mathcal{F}_{n}^{(k)}(x)$ satisfy the following recurrence for $n \geqslant k$

$$
\mathcal{F}_{n}^{(k)}(x)=\bar{p}_{1}(x) \mathcal{F}_{n-1}^{(k)}(x)+\bar{p}_{2}(x) \mathcal{F}_{n-2}^{(k)}(x)+\cdots+\bar{p}_{k}(x) \mathcal{F}_{n-k}^{(k)}(x),
$$

where $\mathcal{F}_{0}^{(k)}(x)=1, \mathcal{F}_{i}^{(k)}(x)=0$ for $i=-1,-2, \ldots,-(k-1)$.

Proof. The identity follows from Equation (11).

We can write the polynomials $\mathcal{F}_{n}^{(k)}(x)$ as a Binet-like formula [14], i.e.,

$$
\mathcal{F}_{n}^{(k)}(x)=\sum_{i=1}^{k} \frac{r_{i, k}(x)^{n+k}}{\prod_{l=1, l \neq i}^{k}\left(r_{i, k}(x)-r_{j, k}(x)\right)},
$$

where $r_{i, k}(x)$ are the roots of the characteristic equation of (12).

Definition 26. The generalized $m$-bonacci-Lucas polynomials are defined by

$$
\mathcal{L}_{n}^{(m)}(x)=r_{1, m}(x)^{n}+r_{2, m}(x)^{n}+\cdots+r_{m, m}(x)^{n} .
$$

Note that

$$
\begin{aligned}
\mathcal{L}_{n}^{(m)}(x) & =\bar{p}_{1}(x) \mathcal{F}_{n-1}^{(m)}(x)+2 \bar{p}_{2}(x) \mathcal{F}_{n-2}^{(m)}(x)+\cdots+m \bar{p}_{m}(x) \mathcal{F}_{n-m}^{(m)}(x), \\
& =\mathcal{F}_{n}^{(m)}(x)+\bar{p}_{2}(x) \mathcal{F}_{n-2}^{(m)}(x)+\cdots+(m-1) \bar{p}_{m}(x) \mathcal{F}_{n-m}^{(m)}(x) .
\end{aligned}
$$

For example, if $m=4$ we obtain the tetrabonacci-Lucas polynomials. The first few terms of this sequence are

$$
4, p_{1}, p_{1}^{2}+2 p_{2}, p_{1}^{3}+3 p_{2} p_{1}+3 p_{3}, p_{1}^{4}+4 p_{2} p_{1}^{2}+4 p_{3} p_{1}+2 p_{2}^{2}+4 p_{4}, \ldots,
$$

where $p_{i}=\bar{p}_{i}(x), i=1,2,3,4$. If $p_{i}=1, i=1,2,3,4$, we obtain the sequence A073817. 
Proposition 27. The generating function of the m-bonacci-Lucas polynomials is

$$
\mathcal{L}_{m}(z):=\sum_{i=0}^{\infty} \mathcal{L}_{i}^{(m)}(x) z^{i}=\frac{m-(m-1) \bar{p}_{1}(x) z-(m-2) \bar{p}_{2}(x) z^{2}-\cdots-\bar{p}_{m-1}(x) z^{m-1}}{1-\bar{p}_{1}(x) z-\bar{p}_{2}(x) z^{2}-\cdots-\bar{p}_{m}(x) z^{m}} .
$$

Proof. The proof runs like in Proposition 4.

\subsection{Combinatorial Interpretation}

Theorem 28. For any polynomial $\mathcal{F}_{n}^{(m)}(x)$ and $\mathcal{L}_{n}^{(m)}(x)$, we have

(i) $\mathcal{F}_{n}^{(m)}(x)=\sum_{k=0}^{n} \omega_{n, k}^{(m)}(x)$ for $n \geqslant 0$,

(ii) For $n \geqslant m$,

$$
L s_{n}^{(m)}(x)=\bar{p}_{1}(x) \sum_{k=0}^{n-1} \omega_{n-1, k}^{(m)}(x)+2 \bar{p}_{2}(x) \sum_{k=0}^{n-2} \omega_{n-2, k}^{(m)}(x)+\cdots+m \bar{p}_{m}(x) \sum_{k=0}^{n-m} \omega_{n-m, k}^{(m)}(x) .
$$

where $\omega_{n, k}^{(m)}(x):=\omega_{n, k}^{(m)}=h_{n, k}^{(m)}=\mathcal{D}_{m}(n-k, k)$ is the sum of weights of $\vec{A}_{m+1}$-weighted paths from $(0,0)$ to $(k, n-k)$ with step set

$$
S_{m}=\left\{H=(0,0), V=(0,1), D_{1}=(1,1), D_{2}=(1,2), \ldots, D_{m-1}=(1, m-1)\right\},
$$

such that $a_{1}(x)+a_{2}(x)=\bar{p}_{1}(x)$, and $a_{i}(x)=\bar{p}_{i-1}(x)$ for $i=1, \ldots, m+1$.

Example 29. The tetrabonacci numbers are defined by the recurrence relation:

$$
\begin{aligned}
& l_{0}=1, \quad l_{1}=1, \quad l_{2}=2, \quad l_{3}=4 \\
& l_{n+4}=l_{n+3}+l_{n+2}+l_{n+1}+l_{n}, \quad \text { for } n \geqslant 0 .
\end{aligned}
$$

The first few terms of the tetrabonacci number are 1, 1, 2, 4, 8, 15, 29, 56, 108, 208, $401, \ldots$, (sequence A000078). The tetrabonacci polynomials $R_{n}(x)$ are defined by the recurrence relation

$$
\begin{gathered}
R_{0}(x) 1, \quad R_{1}(x)=x^{3}, \quad R_{2}(x)=x^{6}+x^{2}, \quad R_{3}(x)=x^{9}+2 x^{5}+x, \\
R_{n+4}(x)=x^{3} R_{n+3}(x)+x^{2} R_{n+2}(x)+x R_{n+1}(x)+R_{n}(x), \text { for } n \geqslant 0 .
\end{gathered}
$$

The generating function of the tetrabonacci polynomials is

$$
\sum_{n=0}^{\infty} R_{n}(x) z^{n}=\frac{1}{1-x^{3} z-x^{2} z^{2}-x z^{3}-z^{4}} .
$$

If $a_{1}(x)=x^{3}, a_{2}(x)=0, a_{3}(x)=x^{2}, a_{4}(x)=x$ and $a_{5}(x)=1$ in (3), we obtain the tetrabonacci polynomials from the row sums of the Riordan array $\mathcal{H}_{4}\left(x^{3}, 0, x^{2}, x, 1\right)$.

From Theorem 14- $(i)$, we obtain a combinatorial interpretation of $R_{n}(x)$. For example,

$$
R_{3}(x)=x^{9}+2 x^{5}+x=\sum_{k=0}^{3} \omega_{3, k}^{(4)}=0+x+2 x^{5}+x^{9} .
$$

In Figure 4, we show the corresponding weighted lattice paths. 


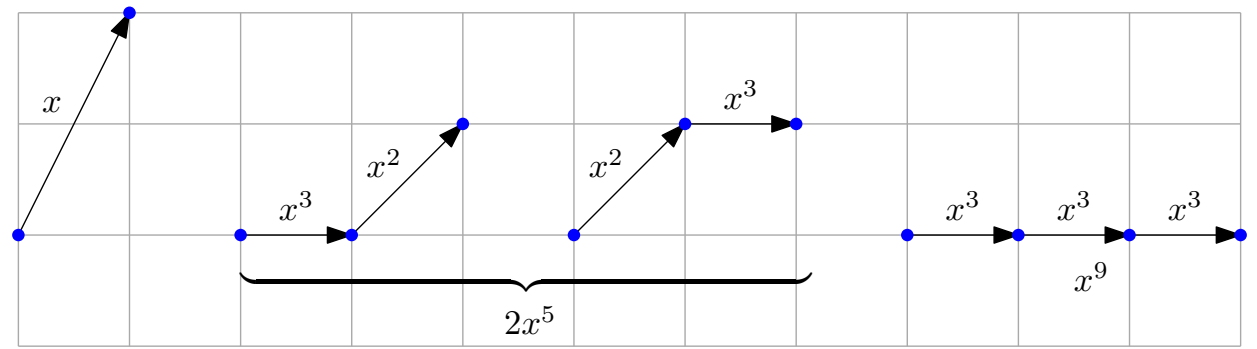

Figure 4: $\left(x^{3}, x^{2}, 0, x, 1\right)$-weighted paths and tretranacci polynomials.

\section{Acknowledgements}

We would like to thank the anonymous referee for valuable comments to improve the final version. The second author thanks the invitation to Bogotá where the mayor part of this paper was done.

\section{References}

[1] C. Banderier and S. Schwer. Why Delannoy numbers? J. Statist. Plann. Inference, 135:40-50, 2005.

[2] P. Barry. On integer-sequence-based constructions of generalized Pascal triangles. $J$. Integer Seq., 9 Article 06.2.4, 2006.

[3] P. Barry. On the central coefficients of Riordan matrices. J. Integer Seq., 16 Article 13.5.1, 2013.

[4] F. Bernhart. Catalan, Motzkin, and Riordan numbers. Discrete Math., 204(1-3): 73112, 1999.

[5] B. Birregah, P. K. Dohb and K. H. Adjallah. A systematic approach to matrix forms of the Pascal triangle: The twelve triangular matrix forms and relations. European J. Combin., 31:1205-1216, 2010.

[6] R. Brawer and M. Pirovino. The linear algebra of the Pascal matrix. Linear Algebra Appl., 174:13-23, 1992.

[7] G. S. Call and D. J. Velleman. Pascal's matrices. Amer. Math. Monthly, 100: 372376, 1993.

[8] D. Callan. On generating functions involving the square root of a quadratic polynomial. J. Integer Seq., 10 Article 07.5.2, 2007.

[9] G.-S. Cheon, H. Kim and L. W. Shapiro. A generalization of Lucas polynomial sequence. Discrete Appl. Math. 157: 920-927, 2009.

[10] L. Comtet. Advanced combinatorics. D. Reidel Publishing Company, 1970.

[11] M. Dziemiańczuk. Counting lattice paths with four types of steps. Graphs Combin., 30:1427-1452, 2014. 
[12] A. Edelman and G. Strang. Pascal Matrices. Amer. Math. Monthly, 111(3): 361-385, 2004.

[13] L. Ericksen. Lattice path combinatorics for multiple product identities. J. Statist. Plann. Inference, 140: 2213-2226, 2010.

[14] I. Flores. Direct calculation of $k$-generalized Fibonacci numbers. Fibonacci Quart., 5(3): 259-266, 1967.

[15] R. D. Fray and D. P. Roselle. Weighted lattice paths. Pacific J. Math., 37(1): 85-96, 1971.

[16] V. E. Hoggatt Jr. and M. Bicknell. Generalized Fibonacci polynomials. Fibonacci Quart., 11: 457-465, 1973.

[17] E. Kiliç and H. Prodinger. Some double binomial sums related with the Fibonacci, Pell and generalized order- $k$ Fibonacci numbers. Rocky Mountain J. Math., 43(3): 975-987, 2013.

[18] D. Merlini, D. G. Rogers, R. Sprugnoli, M- Cecilia Verri. Underdiagonal lattice paths with unrestricted steps. Discrete Appl. Math., 91(1-3): 197-213, 1999.

[19] L. W. Shapiro, S. Getu, W. Woan and L. Woodson. The Riordan group. Discrete Appl. Math., 34: 229-239, 1991.

[20] N. J. A. Sloane. The On-Line Encyclopedia of Integer Sequences. https://oeis.org/.

[21] R. Sprugnoli. Riordan arrays and combinatorial sums. Discrete Math., 132: 267-290, 1994.

[22] R. Sulanke. Objects counted by the central Delannoy numbers. J. Integer Seq. 6 Article 03.1.5, 2003.

[23] Y. Yang and C. Micek. Generalized Pascal functional matrix and its applications. Linear Algebra Appl. 423: 230-245, 2007.

[24] Z. Zhang. The linear algebra of the generalized Pascal matrix. Linear Algebra Appl. 250: 51-60, 1997.

[25] Z. Zhang and X.Wang. A factorization of the symmetric Pascal matrix involving the Fibonacci matrix. Discrete Appl. Math. 155: 2371-2376, 2007. 